\title{
Derivative and Integration on Time Scale with Mathematica
}

\author{
Ahmet Yantir \\ Department of Mathematics, Izmir Institute of Mathematics, \\ Turkey \\ ahmetyantir@iyte.edu.tr
}

\begin{abstract}
1 Abstract
Mathematical modelling of time dependent systems is always interesting for applied mathematicians. First continuous and then discrete mathematical models were built in the mathematical development from ancient to modern times. With the discovery of time scale, the problem of irregular systems was solved in the 1990s. In this paper we explain the derivative and integral of functions of time scales and the solution of some basic calculus problems using Mathematica.
\end{abstract}

\section{Introduction}

In section 2, we define time scale and the forward and backward jump operators which are used in the definition of time scale and derivative. We also give Mathematica commands which find the values of forward operators at given points. In section 3 , we give the definition and some important theorems on the time scale derivative. In this paper we deal with only the forward difference derivative on time scales. We give Mathematica commands in two cases: first for given time scales which are the combination of discrete and continuous sets, and then for discrete cases with a rule given by the user. In section 4, we present the integration on time scales with Mathematica in two different Modules, one for purely discrete cases and one for a combination of discrete and continuous cases.

\section{$3 \quad$ What is Time Scale?}

Let $\mathrm{T}$ be any closed subset of the real numbers, $\mathrm{R}$. $\mathrm{T}$ is called a time scale. 


\section{Example 3.1}

The real numbers, the integers, the natural numbers, and nonnegative integers are examples of time scales, as are

$$
\begin{aligned}
\text { In }[1]:=T 1 & =0 \leq x \leq 3 \| x=4|| x=9 ; \\
T 2 & =\text { Table }\left[2^{\wedge} n,\{n, 0,10\}\right]
\end{aligned}
$$

The rationals, the irrationals, complex numbers, and the open intervals of real numbers are not time scales.

In order to define the derivative on time scale we need to define backward and forward jump operators which are used in the definition of time scale derivative. [2]

\section{Definition 3.1}

Let $\mathrm{T}$ be a time scale. For $\mathrm{t} \in \mathrm{T} ; \mathrm{t}<\mathrm{MaxT}$; where MaxT is a real number, we define the forward jump operator $\sigma: \mathrm{T} \rightarrow \mathrm{T}$ by $\sigma(\mathrm{t})=\operatorname{Inf}\{\mathrm{s}: \mathrm{s} \in \mathrm{t} ; \mathrm{s}>\mathrm{t}\}$.

We define this operator in Mathematica as follows [2,3,4]:

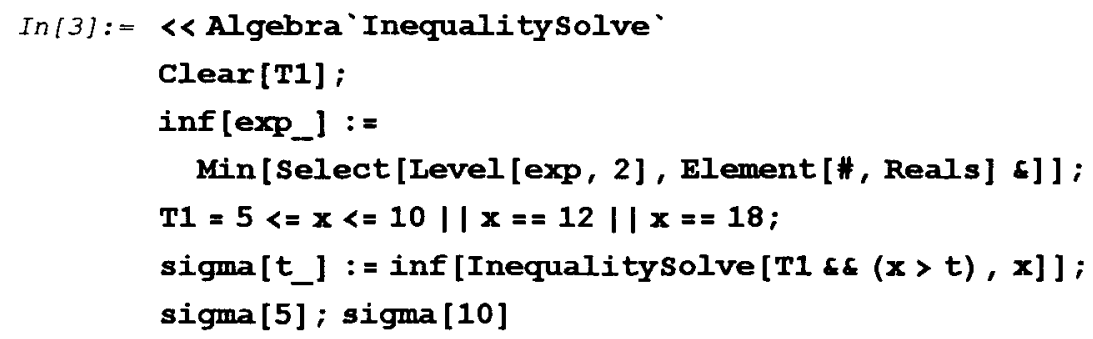

If $\mathrm{T}=\mathrm{R}$, then $\sigma(\mathrm{t})=\mathrm{t}$. If $\mathrm{T}=\mathrm{Z}$ (the integers), then $\sigma(\mathrm{t})=\mathrm{t}+1$. Also $\sigma(\mathrm{MaxT})=\operatorname{MaxT}$.

\section{Definition 3.2}

$\mathrm{t}$ is called a right-dense point if $\sigma(\mathrm{t})=\mathrm{t}$, and right-scattered if $\sigma(\mathrm{t})>\mathrm{t}$.

\section{Definition 3.3}

Let $\mathrm{T}$ be a time scale. For $\mathrm{t} \in \mathrm{T} ; \mathrm{t}>\mathrm{MinT}$; we define backward jump operator $\varrho: \mathrm{T} \rightarrow$ $\mathrm{T}$ by $\varrho(\mathrm{t})=\operatorname{Sup}\{\mathrm{s}: \mathrm{s} \in \mathrm{t} ; \mathrm{s}<\mathrm{t}\}$.

$t$ is called a left-dense point if $\varrho(t)=t$, and left-scattered if $\varrho(t)<t$. 


\section{Derivative on Time Scale}

In order to define the derivative on time scales we need the set below which is derived from the time scale $\mathrm{T}$ as follows:

Definition 4.1

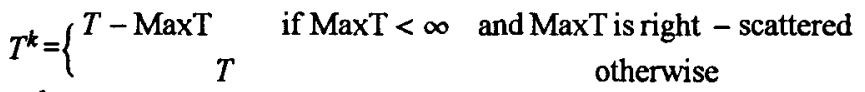

$T^{k}$ is called the region of differentiability.

\section{Definition 4.2}

Let $\mathrm{f}: \mathrm{T} \rightarrow \mathrm{R}$ be a function and $\mathrm{t} \in T^{k}$. If there exist a neighborhood $U_{t}$ such that $|\mathrm{f}(\sigma(\mathrm{t}))-\mathrm{f}(\mathrm{s})-\mathrm{a}[\sigma(\mathrm{t})-\mathrm{s}]| \leq \epsilon|\sigma(\mathrm{t})-\mathrm{t}|$

is satisfied for all $\mathrm{s} \in U_{t}, \epsilon>0$ then $\mathrm{f}$ is $\Delta$ differentiable at the point $\mathrm{t}$. $\mathrm{a}$ is called $\Delta$ derivative of $f$ at the point $t$.

$\mathrm{a}=f^{\Delta}(t)=\lim _{s \rightarrow t} \frac{f(\sigma(t))-f(s)}{\sigma(t)-s}$.

We give an example of the $\Delta$ derivative in Mathematica as follows:

Example 4.1

Let $\mathrm{f}(\mathrm{x})=x^{2}$ and $\mathrm{g}(\mathrm{x})=x^{3}+x^{2}+1$. Let $\mathrm{T} 4=[5,10] \cup\{12,18\} \bigcup[20,22]$. Find $\left(\frac{f}{g}\right)^{\Delta}(10)$.

\section{Solution:}

By definition 4.2

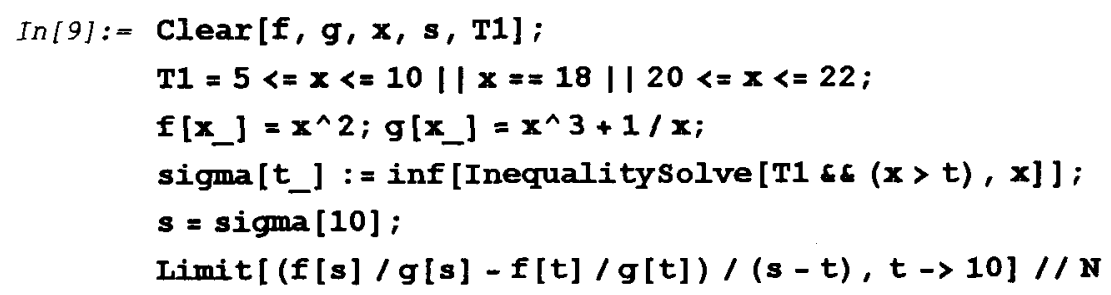

So we get $\left(\frac{f}{g}\right)^{\Delta}(10)=-0.00555437[2,3,4]$ 


\section{Theorem 4.1}

Let $\mathrm{f}, \mathrm{g}: \mathrm{T} \rightarrow \mathrm{R}$ be functions and $\mathrm{t} \in T^{k}$. If $\mathrm{f}$ and $\mathrm{g}$ have a $\Delta$ derivative at $\mathrm{t}$ then 1) $\mathrm{f} g$ has a $\Delta$ derivative at $\mathrm{t}$ and $(f g)^{\Delta}(\mathrm{t})=f^{\Delta}(t) g(\mathrm{t})+\mathrm{f}(\sigma(\mathrm{t})) g^{\Delta}(\mathrm{t})=f(t) g^{\Delta}(\mathrm{t})+f^{\Delta}(t) g(\sigma(\mathrm{t}))$ 2) If $\mathrm{g}(\mathrm{t}) \mathrm{g}(\sigma(\mathrm{t})) \neq 0$ then $\frac{f}{g}$ has a $\Delta$ derivative at $\mathrm{t}$ and $\left(\frac{f}{g}\right)^{\Delta}(\mathrm{t})=\frac{f^{\Delta}(t) g(t)-f(t) g^{\Delta}(t)}{g(t) g(\sigma(t))}$.

\section{Example 4.2}

[1] has a 'paper' proof of this theorem. We found the value of the derivative of $\mathrm{f} / \mathrm{g}$ in example 4.1 by using definition 4.2 . We can use Theorem 4.1 to compute the derivative of $f / g[2,3,4]$ :

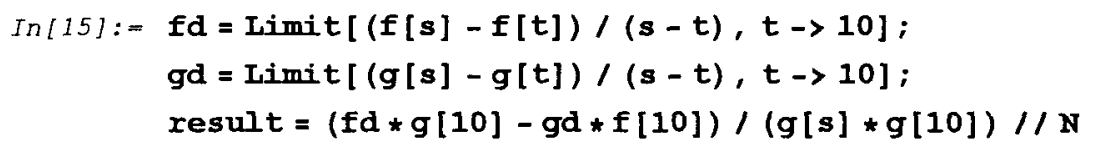

We define the derivative for purely discrete time scales in Mathematica as follows $[3,4]$ :

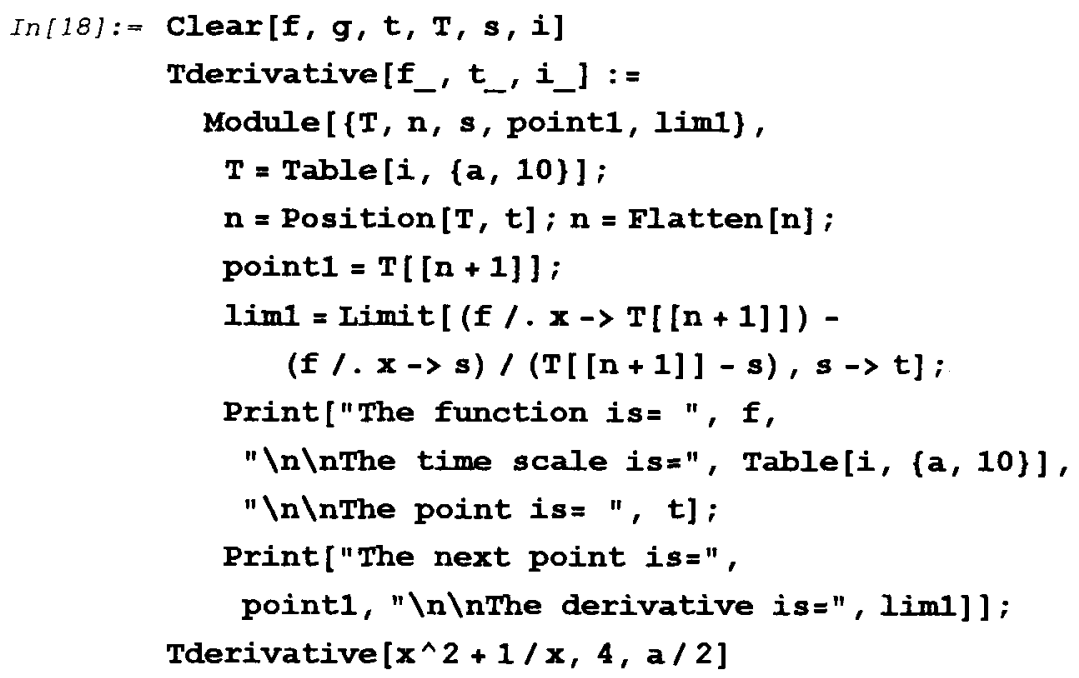




\section{Integration}

\section{Definition 5.1}

A function $\mathrm{f}: \mathrm{T} \rightarrow \mathrm{R}$ is called regulated if it has finite right-sided limits at all right-dense points in $T$, and has finite left-sided limits at all left-dense points in $T$. A function $\mathrm{f}: \mathrm{T} \rightarrow \mathrm{R}$ is called rd-continuous if it is continuous at all right-dense points in $\mathrm{T}$ and its leftsided limits exist (and is finite) at all left-dense points in $\mathrm{T}$. The set of rd-continuous functions in $\mathrm{T}$ is denoted by $C_{\mathrm{rd}}$.

If $F^{\Delta}(t)=\mathrm{f}(\mathrm{t})$ is satisfied for all $\mathrm{t} \in T^{k}$, then $\mathrm{F}$ is called the anti-derivative of $\mathrm{f}$.

\section{Theorem 5.1}

Let $a, b \in T$ and $f \in C_{r d}$. If $[a, b]$ consists of only isolated points, then

$$
\begin{gathered}
\int_{a}^{b} f(t) \Delta \mathrm{t}=\left\{\begin{array}{cc}
\sum_{t \in[a, b)}(\sigma(t)-t) f(t) & \text { if } a<b \\
0 & \text { if } a=b \\
-\sum_{t \in[b, a)}(\sigma(t)-t) f(t) & \text { if } a>b
\end{array}\right. \\
\text { In particular } \int_{t}^{\sigma(t)} f(\tau) \Delta \tau=(\sigma(\mathrm{t})-\mathrm{t}) \mathrm{f}(\mathrm{t})
\end{gathered}
$$

See [1] for a 'paper' proof of this theorem. We define the definite integral for purely discrete time scales in Mathematica as follows [3,4]:

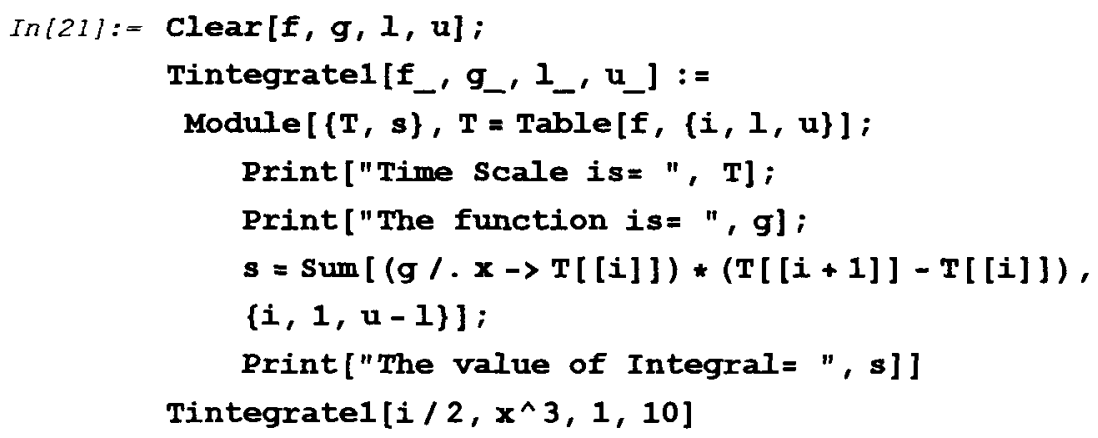

Here $f$ denotes the discrete time scale rule, $g$ denotes the integrand, 1 denotes the lower bound of the integral, $u$ denotes the upper bound of the integral. 
If $T$ is a union of continuous and discrete sets we use another Module to solve the problem.

\section{Example 5.1}

Let $\mathrm{T}=[1,3] \cup\{5\} \cup[6,8] \cup\{12,13\}$ and $\mathrm{f}(\mathrm{t})=t^{3}$. Evaluate $\int_{2}^{12} f(t) \Delta \mathrm{t}$.

\section{Solution:}

We can solve this problem with Mathematica as follows $[3,4]$ :

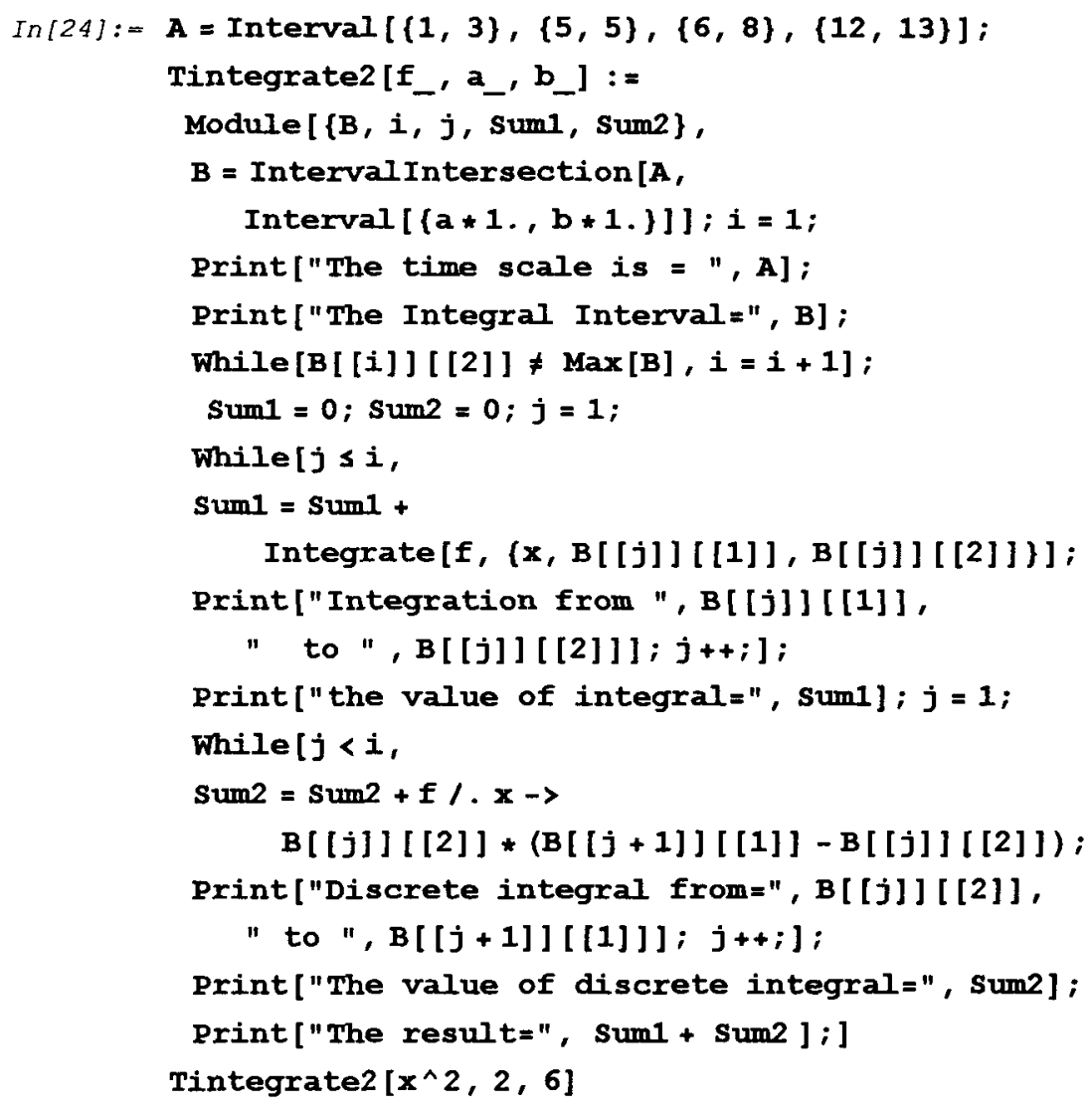




\section{References}

[1] M. Bohner-A. Peterson, Dynamic Equations on Time Scales, 2001, Birkhauser Boston.

[2] A. Yantir-U. Ufuktepe, Basic Calculus on Time Scale, 2003, Lecture Notes on Computer Science (will be published).

[3] J. W. Gray, Mastering Mathematica, 1997, Academic Press.

[4] S. Wolfram, The Mathematica Book, 1996, Cambridge University Press. 